\title{
A large vegetation on a xenopericardial roll elephant trunk graft
}

Sachito Minegishi, MD, PhD, Yusuke Inaba, MD, Hidehito Endo, MD, PhD, and Hiroshi Kubota, MD, PhD, Tokyo, Japan

From the Department of Cardiovascular Surgery, Kyorin University Hospital, Tokyo, Japan. Disclosures: The authors reported no conflicts of interest.

The Journal policy requires editors and reviewers to disclose conflicts of interest and to decline handling or reviewing manuscripts for which they may have a conflict of interest. The editors and reviewers of this article have no conflicts of interest.

Received for publication Oct 29, 2020; accepted for publication Dec 1, 2020; available ahead of print Dec 9, 2020. Address for reprints: Sachito Minegishi, MD, PhD, Department of Cardiovascular Surgery, Kyorin University Hospital, 6-20-2 Shinkawa, Mitaka-shi, Tokyo 181-8611, Japan (E-mail: minegishi@ks.kyorin-u.ac.jp). JTCVS Techniques 2021;6:46-9

2666-2507

Copyright (C) 2020 The Authors. Published by Elsevier Inc. on behalf of The American Association for Thoracic Surgery. This is an open access article under the CC BY-NC-ND license (http://creativecommons.org/licenses/bync-nd/4.0/).

https://doi.org/10.1016/j.xjtc.2020.12.001

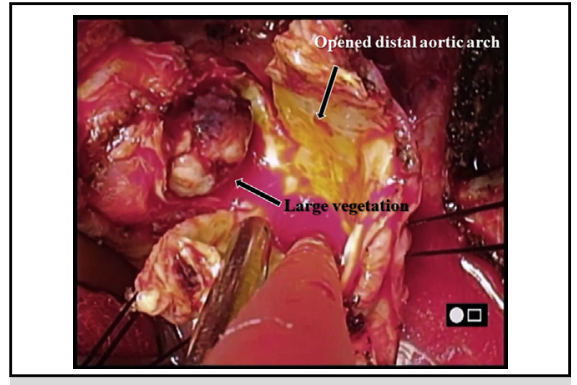

Intraoperative view of the large vegetation in the xenopericardial roll elephant trunk.

CENTRAL MESSAGE

This is the first report showing a large vegetation on an elephant trunk using xenopericardial roll graft that almost totally occludes the lumen during the late postoperative period.

See Commentaries on pages 50 and 52.
Elephant trunk (ET) graft infection is a rare complication. ${ }^{1}$ We present the uncommon case of a large vegetation on an ET, which almost totally occluded the lumen during the late postoperative period.

\section{CLINICAL SUMMARY}

A 72-year-old woman was admitted to our outpatient clinic because of fever and sudden arterial hypertension. She underwent an emergency total aortic arch replacement (TAR) using the frozen ET installation for DeBakey type I acute aortic dissection 7 months before the clinic visit. Two weeks after the TAR, a prosthetic graft infection caused by Enterobacter cloacae occurred, and redo TAR with the ET technique using a xenopericardial roll graft (XRG) and local coverage with the omentum and right pectoralis major muscle flap was performed after drainage, negative pressure wound therapy, and administration of specific intravenous antibiotics. We inserted an XRG as an ET, which had the same diameter as her distal aortic arch. Enhanced computed tomography (CT) scan taken after the redo TAR revealed neither stenosis nor abnormal thrombus on the XRG (Figure 1). Her ankle-brachial pressure index was $>1.0$ bilaterally. Her immune status was not suppressed. Although she remained well 3 months after the redo TAR, she presented to our emergency department with dyspnea and hypotension. Further examination revealed rupture of hepatic abscess, and emergency radiofrequency ablation was performed. Multiple blood cultures taken during the readmission were positive for Candida albicans; therefore, amphotericin B was administered for 2 weeks. Enhanced CT scan taken at the emergency department revealed there was no apparent problem around the XRG. Although she remained well 1 month after the radio-frequency ablation, she presented to our clinic with hypertension and fever. Blood pressure in her upper and lower limbs was 178/95 $\mathrm{mm} \mathrm{Hg}$ and 90/ $60 \mathrm{~mm} \mathrm{Hg}$, respectively. Her body temperature was $38.6^{\circ} \mathrm{C}$. Laboratory tests showed a white blood cell count of $3.500 / \mu \mathrm{L}$, hemoglobin of $9.5 \mathrm{~g} / \mathrm{dL}$, C-reactive protein level of $2.67 \mathrm{mg} / \mathrm{dL}$ (clinic's laboratory reference value $<0.14$ ), and beta-D-glucan of $47.49 \mathrm{pg} / \mathrm{mL}$ (clinic's laboratory reference value $<11$ ). CT angiogram revealed a large thrombus on the ET using XRG that occluded almost of the lumen (Figure 2, $A-C$ ) and infarcts in the liver. The thrombus was $44 \mathrm{~mm}$ in length and $29 \mathrm{~mm}$ in width.

An urgent operation was performed through left thoracotomy. Under deep hypothermic cardiopulmonary bypass, the descending aorta was excised and the giant vegetation was visualized in the ET (Video 1 and Figure 2, D). After 

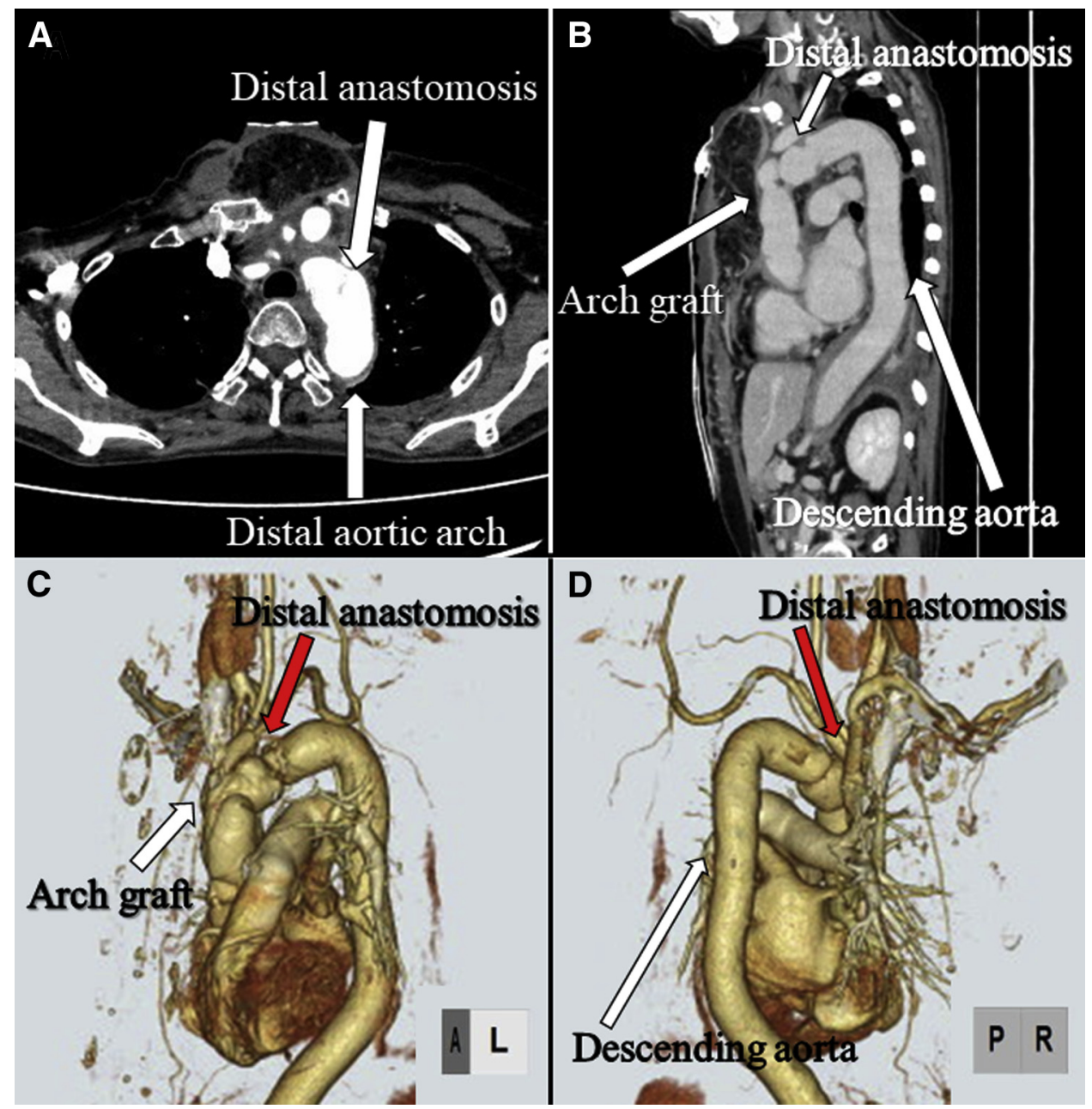

FIGURE 1. Chest computed tomography scan with enhancement in the axial (A) and the sagittal (B) view demonstrates no stenosis or thrombus in the elephant trunk using xenopericardial roll graft. Left (C) and right rear (D) views of a 3-dimensional reconstruction of the dynamic computed tomography scan. There was no apparent problem in total aortic arch replacement using branched xenopericardial roll graft. There was no size discrepancy between the xenopericardial roll elephant trunk graft and native distal aortic arch.

complete removal of the vegetation, the descending aorta was replaced with another XRG. Blood and lesion cultures were positive for $C$ albicans; therefore, intravenous administration of fosfluconazole was continued for 4 weeks and oral fluconazole was administered. She was discharged following full recovery.

Informed consent was obtained from the patient for publication of this case.

\section{DISCUSSION}

Aortic prosthetic infection remains among the most challenging surgical problems. This condition is classically associated with high morbidity and mortality rates, ${ }^{2}$ but the optimal graft material for the reconstruction is still a matter of controversy. ${ }^{3}$ In contrast to abdominal aortic graft infection, ${ }^{4}$ there are no reports of cases of aortic arch graft infection treated with XRG. Our department used branched XRG to replace an infected aortic arch graft. ${ }^{5}$ To the best of our knowledge, this is the first report that shows a vegetation on an ET made with XRG.

A few complications of the ET procedure have been reported, such as kinking or flattening during surgery during the early postoperative period. However, there is no report of ET obstruction during the chronic postoperative phase. There are some case reports of small vegetations being attached to an ET using prosthetic graft resulting in multiple small emboli. ${ }^{1}$ There is no report that presents such a large vegetation. The vena contracta caused by size discrepancy between an ET and native distal arch is considered to be a cause of ET infection, ${ }^{1}$ but this theory may not be applicable to our case because there was no size mismatch (Figure 1). Our ET was made with XRG, which is softer than prosthetic grafts. This may account for this patient's unconventional event.

In the present case, hypertension occurred suddenly. The giant abscess is believed to have formed in a few 

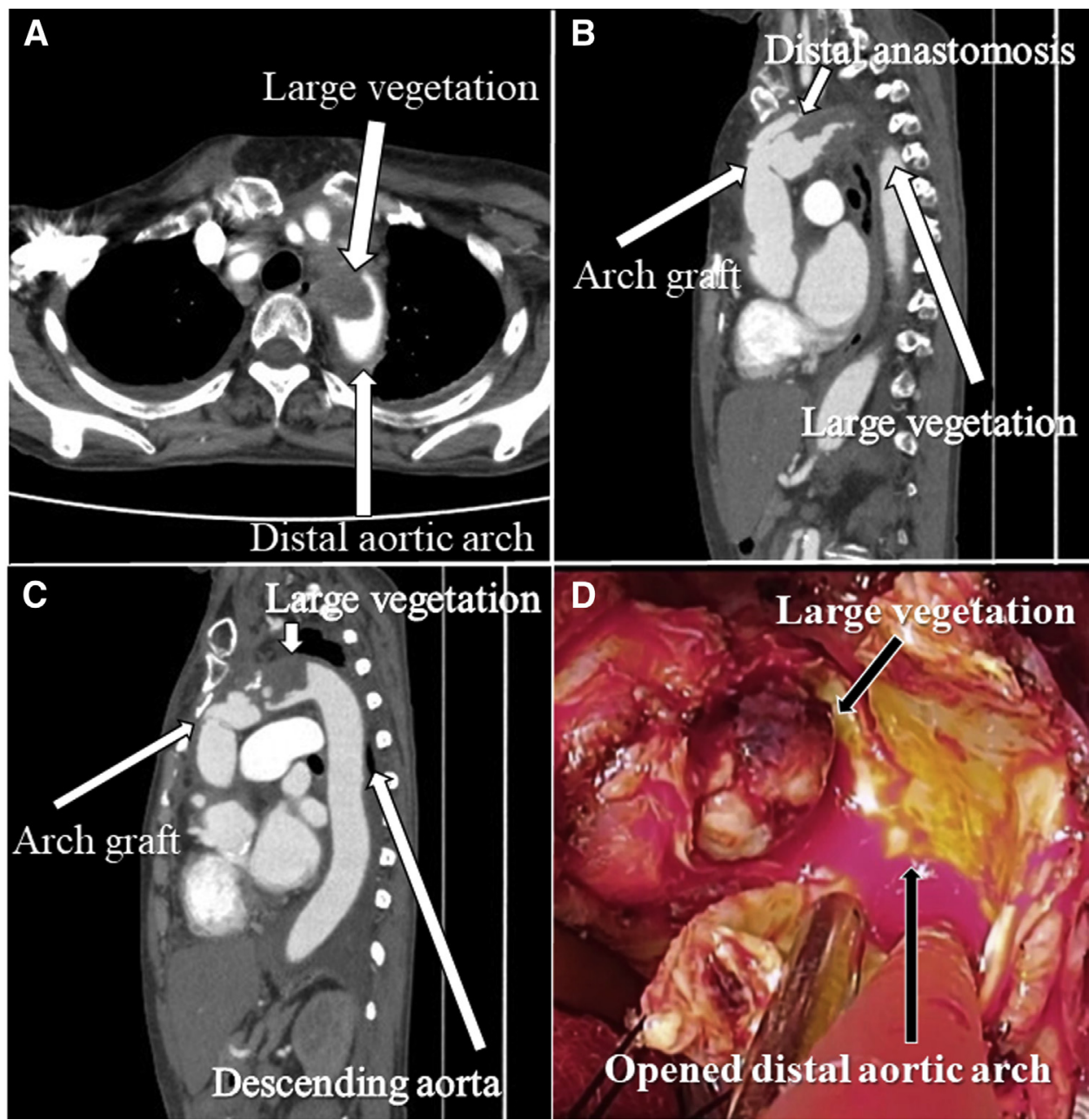

FIGURE 2. Chest computed tomography scan with enhancement in the axial (A) and the sagittal (B and C) view demonstrates the large vegetation in the elephant trunk using xenopericardial roll graft, which almost occluded the lumen of the elephant trunk. Intraoperative view (D) of the large vegetation in the xenopericardial roll elephant trunk graft and the lumen of the opened descending aorta.

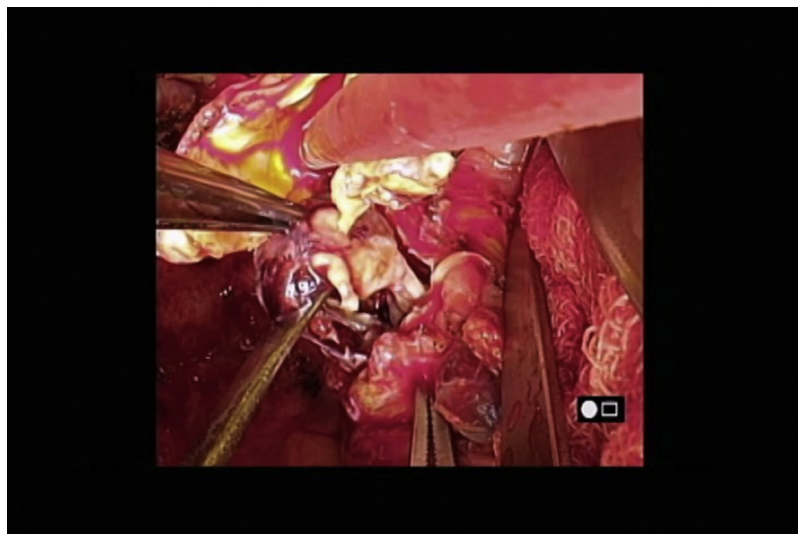

VIDEO 1. Under deep hypothermic cardiopulmonary bypass through left thoracotomy, the descending aorta was excised and the giant vegetation was removed. Video available at: https://www.jtcvs.org/article/S26662507(20)30757-4/fulltext. weeks. Patients with a surgical history of TAR using XRG as an ET, especially in a patient in whom sepsis is suspected, should be monitored for acute abscess formation.

\section{CONCLUSIONS}

This case showed an unusual ET occlusion after TAR using XRG. ET graft occlusion should be taken into consideration when dealing with patients with a surgical history of TAR using XRG.

\section{References}

1. Tanaka A, Sakamoto T, Okada K, Okita Y. Vegetation attached to the elephant trunk. Eur J Cardiothorac Surg. 2013;44:565-6.

2. Coselli JS, Köksoy C, LeMaire SA. Management of thoracic aortic graft infections. Ann Thorac Surg. 1999;67:1990-3.

3. Chakfé N, Diener H, Lejay A, Assadian O, Berard X, Caillon J. European Society for Vascular Surgery (ESVS) 2020 clinical practice guidelines on the management of vascular graft and endograft infections. Eur J Vasc Endovasc Surg. 2020;59: 339-84. 
4. Weiss S, Tobler EL, von Tengg-Kobligk H, Makaloski V, Becker D, Carrel TP, et al. Self-made xeno-pericardial aortic tubes to treat native and aortic graft infections. Eur J Vasc Endovasc Surg. 2017;54:646-52.
5. Kubota H, Endo H, Ishii H, Tsuchiya H, Ohura N, Takahashi Y. Branched xenopericardial roll graft replacement of an infected aortic arch graft. J Card Surg. 2019;34:31-4. 